\title{
THE 50-MOVE RULE REVISITED
}

\begin{abstract}
The Editors
As promised in ICCA Journal, Vol. 12, No. 2, p. 123 the 50-move rule is a subject to be pursued because of its essential nature to our readership. The previous history of the rule is well-known: it was almost absolute up to 1983 (KNNKP being the only exception allowing 100 moves and, in practice, KRBKR to be decided by tournament). In 1983, Thessaloniki saw a confirmation of a prior meeting of the FIDE Rules Commission by the FIDE Congress.
\end{abstract}

Its upshot was that all exceptions to the 50-move rule were explicitated to three endgames only: KNNKP, $\mathrm{KRBKR}$ and $\mathrm{KRP}(\mathrm{a} 2) \mathrm{KbBP}(\mathrm{a} 3)$, for which a uniform maximum of 100 moves was established. Moreover, the extension to 100 moves for KNNKP was only permissible for stated positions of the Pawns and one of the Knights.

As we see it, computer-based results, firmly founded on exhaustively enumerated databases for five- and sixmen endings, have greatly perturbed the chess world. It is due to Dr. B.M. Kažić, the President of the FIDE Rules Commission, that the matter has been broached at all. As a result of a particularly laborious project the articles 10.8 and 10.9 , both dealing with the fifty-move rule, were reconsidered and eventually voted upon during the November 22, 1988 meeting. It is notable that no fewer than 5 submissions were received, indicating that agreement was difficult to obtain.

Proposals varied from deleting all exceptions to the fifty-move rule to a highly-qualified extension of the rule in certain highly-specific cases. One of the proposals eventually rejected was for the arbiter to announce, ahead of a tournament or match, what extensions he would allow in what positions, the arbiter thus been given complete freedom and implying different rules for different tournaments. The idea, we add editorially, seems to have been that this formula would allow arbiters to adapt their rulings in the light of the latest results, presumably to be obtained by computer investigation.

In the end, the 50-move rule of article 10.8 was confirmed, exceptions and all, these exceptions to be recorded in article 10.9, now reading:

"The number of 50 moves mentioned in Article 10.8 will be extended to 75 moves for the following positions:

(a) king, rook and bishop against king and rook.

(b) king and two knights against king and pawn.

(c) king, queen and a pawn one square from promotion against king and queen.

(d) king and queen against king and two knights.

(e) king and queen against king and two bishops.

(f) king and two bishops against king and knight."

[Editorial comment: The Editors are most grateful to Dr. B.M. Kažić for providing them with the minutes of the FIDE Rules Commission and wish to recognize their indebtednes for his cooperation.

Still, the Editors insist on the introduction of a $k$-move rule (cf. Dekker et al., 1989), independently of the configuration on the board. We are aware that, in so proposing, we alter the conventions of human chess as now practised. However, in altering them, the proponents feel that they do not adulterate the game; rather, they contribute to a trend towards making it more interesting and more competitive.]

\section{REFERENCE}

Dekker, S.T., Herik, H.J. van den and Herschberg, I.S. (1989). Perfect Knowledge and Beyond. Advances in Computer Chess 5 (Ed. D.F. Beal), pp. 295-312, North-Holland, Amsterdam. 